\title{
MR On-SeT: A Mixed Reality Occupational Health and Safety Training for World-Wide Distribution
}

https://doi.org/10.3991/ijet.v16i05.19661

\author{
Sebastian Felix Rauh $\left({ }^{\varpi}\right)$, Marius Koller, Philip Schäfer, Gerrit Meixner \\ UniTyLab, Heilbronn University, Heilbronn, Germany \\ sebastian.rauhehs-heilbronn.de \\ Cristian Bogdan, Olga Viberg \\ Royal Institute of Technology KTH, Stockholm, Sweden
}

\begin{abstract}
The current generation of dedicated Mixed Reality (MR) devices can be considered as the first generation, which is truly mobile while also being capable of sufficient tracking and rendering. These improvements offer new opportunities for the on-set use of MR devices enabling new ways of using MR. However, these new use cases raise challenges for the design and orchestration of MR applications as well as how these new technologies influence their field of application. In this article, we present MR On-SeT, a MR occupational health and safety training application, which is based on the experiences of an operational division of a world-wide operating German company. The intended purpose of MR On-SeT is to increase employees' awareness of potential hazards at industrial workplaces by using it in occupational health and safety training sessions. Since the application is used at various locations throughout the company's world-wide subsidiaries, we were able to evaluate it through an expert survey with the occupational health and safety managers of seven plants in France, Germany, Japan, and Romania. They reported the condensed experience of around 540 training sessions collected within three months. The purpose of the evaluation was twofold: 1. to understand their perceived attitudes towards the application-in-use, and 2. to collect feedback they received from respondents in training sessions. The results suggest that MR On-SeT can be used to extend current, predominantly theoretical, methods of teaching occupational health and safety at work, which also motivates experienced employees to actively engage in the training sessions. Based on the findings, several design implications are proposed.
\end{abstract}

Keywords-Mixed Reality, Occupational Health and Safety Training, Workplace learning, Design Implications

\section{Introduction}

Research on Mixed Reality (MR; including Augmented Reality, AR) and Virtual Reality (VR) in education and training has been growing exponentially over the past 20 years [1]. These technologies are now available for the mainstream and consumer 
market. However, it is important to differentiate between them: whereas MR consists of the combination of real and virtual content (e.g., the integration of virtual objects to enhance the user's real physical environment), VR provides the ability to cut out the physical world and immerse users in a completely computer-generated environment [2]. According to a survey by the World Economic Forum [3], 53\% of the companies in the professional services are likely to adopt both technologies before 2022. The benefits, such as increased motivation to actively engage in the training or the availability of new modes of interaction, of using MR and VR for training and education have been validated in different studies over the past years (see e.g., [4]). Also, both technologies have been used in various training use-cases, e.g., in health training [5], at universities for hybrid education [6], in a training approach for OHS [7] or in teacher training in a MR integrated learning environment [8].

According to the World Health Organization, occupational health and safety (OHS) training aims to develop, acquire, and extend the knowledge and skills required to perform work in a safe manner, while having a positive impact on the sensed safety climate, i.e., the perceived value of safety in a work environment [9], of the organisation [10]. As today, many factors have been identified as important components of a safety climate, including the management and organisational practices, such as adequacy of training and provision of safety equipment [9]. Furthermore, establishing a safety climate is positively perceived by the employees, because the organisation values their safety and well-being [11].

Lecture-based OHS training (e.g., explaining risks supported by depictions of hazardous situations) still are widely adopted, but Lawson et al. [12] have shown that using VR might be more efficient. They for example, could find a higher knowledge retention and engagement levels for VR fire safety training compared to using PowerPoint.

The present study is based on the hypothesis that MR and VR may provide a novel, more engaging approach for training employees on the risks associated with their regular work tasks. Based on our experience, we imply that modern devices such as the Microsoft HoloLens - used in this study - can enable mobile hands-free MR experience among users.

In the next sections, we present related work, followed by an introduction of $M R$ $\mathrm{On}-\mathrm{SeT}$, a MR based OHS training we developed. Afterwards, results of an expert survey conducted directly after the roll-out of $M R O n-S e T$ in seven plants in four countries are presented. In the next section, we discuss implications for designing a MR technology assisted approach to train occupation safety. Finally, conclusions and an outlook on future work are proposed.

\section{Background}

Mixed Reality (MR) describes a continuum between real world and Virtual Reality (VR) [13]. It includes any kind of simulation, in which virtual elements and the real world are blended into a semi-virtualised, mixed environment. Augmented Reality 
(AR, i.e., reality enhanced with virtual objects) and Augmented Virtuality (AV, i.e., VR enhanced with real objects) are part of MR.

Orchestration of MR applications can be defined as the activities necessary to prepare the virtual content and the user experience in such mixed or virtual environments, and to adapt them during run-time [14]. Koller and Rauh et al. [15] distinguish between two types of orchestration: pre-orchestration and live-orchestration. Preorchestration refers to the preparative measures for a user-experience, such as planning the course of action of a specific training session before hand. Many MR and VR training systems are completely pre-orchestrated (e.g., [7]), since the program flow is fixed by design. Live-orchestration describes all measures available to re-adjust or control the user experience during the immersion, including changing digital content during runtime or asking provocative questions. For example, the temporary take-over of a virtual avatar is exemplified for live-orchestration by Koller et al. [16].

OHS training is restrained by the requirement to not expose trainees and coworkers to health and safety risks [17]. Some risks can be reason enough to not conduct training sessions in real-life settings, as for example, working in great heights [18], in the context of OHS trainings sessions. Besides, OHS training on-site can interfere with the production target [17].

One approach to safely expose trainees to hazardous situations in their context of work is the use of VR learning environments, as it was done by Ke et al. [8]. In this context, scholars have already shown the benefits of using MR and VR technologies for OHS training. Eiris et al. [19] demonstrated how to use $360^{\circ}$ images, augmented with traditional two-dimensional user interface elements to design a training-game on computer screens for construction workers. Trainees have to explore the $360^{\circ}$-sphere and identify potentially hazardous situations. By selecting the right fix from multiple possible fixes, they can solve each situation. The authors found that users value the idea of the software, but also stressed potential for improvement, e.g., to use a more advanced display technology or to apply their approach to other work areas [19]. Also, to train construction-workers, Yabuki et al. [20] proposed a system in which workers on-site can discuss OHS issues with their office-bound supervisors. They employed AR to increase awareness for OHS and minimise unsafe conditions. Others [17] demonstrated the use of a Head Mounted Display (HMD) with an advanced head tracking system for training work in great heights on construction sites. They found that experiencing heights in a game reflecting the work context already allows trainees to accustom themselves to the working conditions and therefore, increases their comfort of working under these circumstances after the training. In contrast to the close-to-reality virtual environment described by [17], Shamsudin et al. [7] developed a comic-like environment. They report that trainees struggle to transfer OHS knowledge learned in VR to real-world situations with their VR solution. Van Wyk and De Villiers [21] offered VR for training miners on occupational safety using gaming mechanisms. The authors describe how they have modelled a mining environment containing several hazards for trainees or virtual co-workers, based on contextual requirements and constraints of the setting.

All the aforementioned use cases typically extend OHS training by adding some gamification elements (integrated into software) to facilitate users' learning. Further- 
more, the presented occupational training simulations either use computer screens or VR-HMDs. The former offers only limited levels of engagement and presence, i.e., the sense of being in the virtual environment [22]. The latter takes the user completely out of the real environment, which can cause the problem of incorporating the trainer into the virtual environment, requiring an extended hardware setup. Especially in highly immersive systems, simulation sickness [23], i.e., malaise while and after being exposed to a simulation (e.g., in VR), occurs. In general, many current VR solutions require a rather complex hardware set-up since they often come with some tracking hardware to be installed besides using the HMD.

There is a broad body of research on digital games to enhance learner motivation [24], mainly by providing extrinsic rewards for quantifiable accomplishments [25]. According to Whitton [26], Learn through Play or Playful Learning for adults is a still emerging field. While there are many similarities to children's play, adults bring assumption and values to the practice of play. Whitton specifies that there is need for establishing trust and relationships for playful learning between group members, since the sole availability of games (and playful activities) alone is not enough [26].

Burke et al. [27] defined three types of engagement in OHS training ranging from least engaging (e.g., lectures) over moderately engaging (e.g., programmed instruction) to most engaging (e.g., hands-on experiences). The authors state that the more engaging a training is designed the better the knowledge acquisition and alongside the reduction in lost time injuries.

\section{Implementation}

In this section, we present our approach for implementing MR On-SeT and provide some contextual information. Furthermore, the application is characterised using user flows from the perspective of the OHS managers and the perspective of the trainees.

\subsection{Approach and context}

$M R \mathrm{On}-\mathrm{SeT}$ is an approach to simulate an informal learning experience in a formal learning setting. It attempts to model 'learning in situations' and systematises 'learning by experience' to allow trainees to connect learnings on OHS to their specific work context as suggested by Burke et al. [36]. MR On-SeT aims to reduce the threshold to approach the topic of OHS. To address issues experienced with a VR safety training prototype, e.g., complexity of setup, higher risk of injury, or simulation sickness, we decided to use MR for this solution.

$M R \mathrm{On}-\mathrm{SeT}$ consists of 55 critical situations throughout six different scenarios, which need to be identified and solved by the users. These hazards have been gathered with an experienced manager of the department of Occupational Safety and Environmental Protection in the Chassis Systems Control division of Robert Bosch $\mathrm{GmbH}$, Abstatt, Germany. This manager collects data on incidents reported by local OHS managers of the world-wide distributed facilities. OHS managers collect work accidents oversee planning and conducting OHS training according to the needs of the 
employees in their plant, past incidents, the companies OHS guidelines, and legal regulations. Furthermore, they instruct OHS officers, who are in charge of putting the concept in place in their specific department and serve as contact person for OHS concerns of their colleagues.

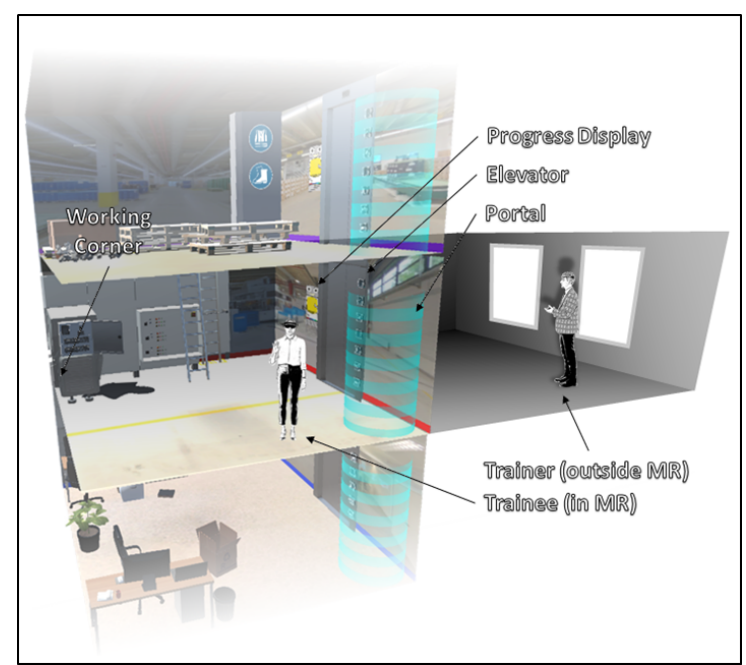

Fig. 1. Concept of the continuous world map of MR On-SeT: The scenes are arranged on several floors in a virtual seven-storey building. The "working corner", marking the half of the cube where safety hazards can be found and the mixed environment is aligned with the physical corner, is in the back left corner and the elements to navigate through the world map are located in the opposite (i.e., front right) corner. Rooms of the following scenes are shown (from bottom to top): Office, Manufacturing (currently active), and Logistics.

Basing the content creation on the manager's experience enabled us to create a solution tailored to nowadays typical work accidents, such as stumbling over an open drawer of an office container or reaching into running machines after bypassing safety shutdown mechanisms. MR On-SeT provides hazardous situations in the following contexts: Electrical Safety, Maintenance, Assembly, Logistics, Manufacturing, and Office. All these scenes have two rooms depicting diverse safety hazards. In the centre of Figure 1, one of two rooms of the maintenance scene is shown, containing three hazardous situations. Furthermore, the initial scene, called Lobby (Figure 2), functions as a three-dimensional main menu, tutorial, and an entrance space.

In this study, we used Microsoft HoloLens 1. It is a binocular MR-HMD equipped with a set of different environment sensing cameras. It weighs 579 gram and has an estimated battery life of two to three hours. The MR application was developed in Unity3D, a multiplatform game engine and development environment. Microsoft provides a MR framework, called MixedReality Toolkit. To select objects on the HoloLens Microsoft defined the 'Air Tap'-Gesture, which relies on the mental model of using the left mouse button (see [28]). To execute this gesture, users have to put the spread-out thumb and index finger together like pinching someone and then release. 


\subsection{User Flow}

In this section we describe how the user flow is orchestrated from the perspective of the OHS mangers and the trainees. Both are primary users of MR On-SeT at different times.

Setting Up MR On-SeT: The set-up of MR On-SeT is performed by the OHS Managers. After switching on the device, they have to consider whether they want to use the streaming option, enabling others (e.g., the OHS managers or co-trainees) to follow the immersed trainee (i.e., the trainee who is wearing the MR-HMD) by streaming the field of view to an additional screen. If they select to enable streaming, the OHS managers have to set-up a WiFi network using their computer or an additional router. Afterwards (or if they choose to not enable streaming), the OHS managers have to start MR On-SeT by clicking on the Tile (icon) in the HoloLens' start menu. The scanning wizard starts, supporting the OHS manager to anchor the mixed environment in one corner of the physical room. This replaces the HoloLens' standard way of placing virtual objects by dragging, rotating and scaling them oneself until they are at the right position in the right orientation and of right size. The OHS managers are assisted by scanning the room in the area of the corner where they want to place the mixed environment and then can place the room by using the pre-defined 'Air-Tap' gesture to place it. After successfully anchoring $M R O n-S e T$ in the physical room, the Lobby is displayed. OHS managers can reset the overall score, displayed behind the front desk (see Figure 2, yellow screen on the right). MR On-SeT is set-up and ready for handing it over to trainees.

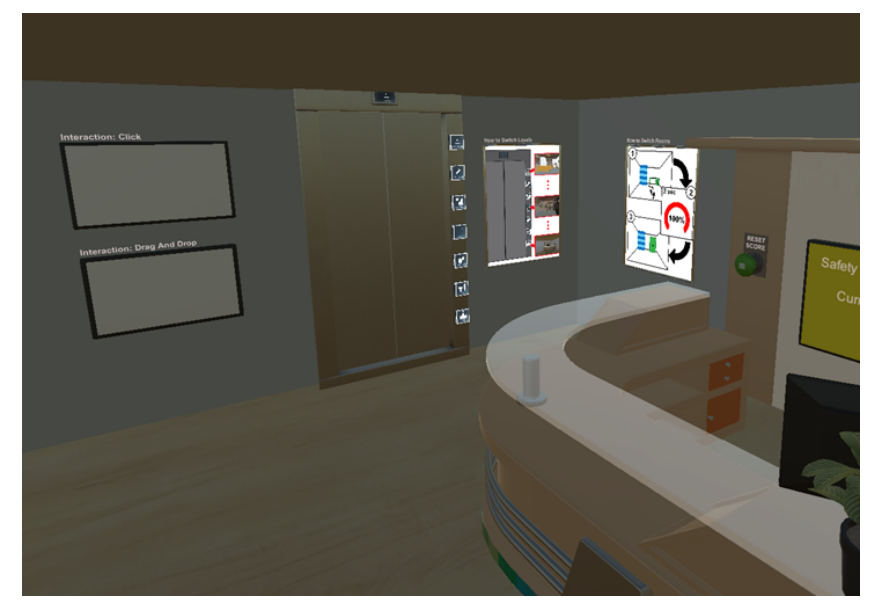

Fig. 2. The Lobby. It serves as three-dimensional main menu, an area to accustom to the interaction concept and welcomes the trainee in the application, like a real lobby of a building. Behind the front desk, a display shows the current score (yellow display on the right). Next to it, the user can reset the score and all changes made. On the left side of the elevator, the user can watch short clips on the monitors, explaining how to use the

Air-Tap gesture to interact within MR On-Set, while on the elevators right side, the general concept of navigating through the mixed environment is explained on two posters. 
Experiencing and solving safety hazards: $M R O n-S e T$ is designed to let trainees orchestrate their workflow (i.e., the order rooms are visited, and hazardous situation are solved) by themselves but also let others (e.g., the OHS managers) intervene.

To access the mixed environment, the trainees have to put on the HMD. In the Lobby, trainees can familiarise themselves with being immersed in MR in general, but also with the 'Air-Tap' gesture and how to use it to drag and drop objects. Short video clips, illustrating these two interaction modes of $M R O n-S e T$, are displayed in two screens (see Figure 2, the two screens on the left). Both interaction modes are based on the 'Air-Tap' gesture. Furthermore, trainees can learn how to navigate through the $M R O n-S e T$ mixed environment on two posters, depicting the use of the elevator to change between scenes, such as Lobby or Logistics, and the use of the portal (see Figure 1, the blue striped cylinder in the front right) to change rooms within on scene. This concept aims to offer the experience of being in a seven-storey building containing different work areas.

Whenever the trainees feel confident to change rooms, or they are triggered by spectators (co-trainees or trainer), they click on one of the buttons on the elevator to move to another storey. All storeys (except the Lobby) display several hazardous situations in one of six work contexts. Trainees can look for these situations by moving through the mixed environment. To solve OHS hazards they either click on an object (e.g., closing an open fuse box lid) or they can remove the hazard causing object by holding and dragging it to a safe location (e.g., moving a ladder, which is blocking an emergency stop). While dragging the objects, users are aided by a hint in form of a hologram depicting the proper location of the object. If streaming is available, trainees in MR might be guided by the OHS managers or even their spectating cotrainees. Trainees can change the room by using the portal and travel to another scene by using the elevator at any time. The number of solved and total hazards of the current room is displayed next to the elevator (Figure 1, robot left to the elevator). This allows the users to keep track of their progress at any time.

\section{$4 \quad$ Expert Survey}

In order to understand OHS managers' perceived attitudes towards the usage of MR On-Set we handed out questionnaires in March 2019 to explore what impact on the context of use of MR On-SeT as mobile MR learning environment has. The participation in the study was voluntary. We received feedback from seven (out of eleven) OHS managers in charge of the OHS of plants located in Japan, Romania, France, and Germany. In their answers they condensed their experience but also the experience of about 540 employees who used MR On-SeT since December 2019.

\subsection{Questionnaire}

Expert surveys have the power to quickly produce results [29]. Besides, we decided to hand out questionnaires to collect data for several reasons: First, we wanted to motivate as many OHS managers as possible to participate. Hence, we aimed to re- 
duce the time needed to participate. Second, this study aims to initially understand the use of MR On-SeT and how the application and the current tools can coexist and benefit from each other. Therefore, we wanted to explore the impact as soon as possible after the roll-out of MR On-SeT to collect data on the changes it caused.

We asked the participating OHS managers to report on their experiences and what they would like to be improved. Also, we asked them to specify the occasion they used MR On-SeT. We explicitly asked the responding OHS managers to not just reflect on their experience, but also to include the feedback they received from trainees during the use of MR On-SeT.

In addition, to better understand the target group of $M R O n-S e T$ we collected demographic data about the trainees in the participating plants.

\subsection{Data analysis}

To identify patterns in the transcribed survey data a reflexive thematic analysis [30] has been performed. We chose to approach the thematic analysis in an inductive way, i.e., we developed themes based on the content of the data. This approach best acknowledges the explorative character of our survey and, therefore, can help to better understand the use of MR On-SeT. Furthermore, we decided to analyse the data using the inductive approach, because this allows to inspect and evaluate the data in an unbiased way. All statements related to one question were clustered. First, after general familiarisation with the data, we clustered the particular statements to preliminary themes (e.g., Orchestration), according to emerging codes. Second, we merged these clusters to overarching themes (e.g., Learn through Play) and reviewed them. The final themes, respondents who contributed statements related to a theme, and some descriptive statements are presented (Table 1) for occasions $M R$ On-SeT has been used. In Table 2, the themes, subthemes and a short description of statements related to questions regarding user experience and potential improvements are presented.

Based on the themes, we suggest implications for designing a MR training system and discuss the needs of trainers and trainees and how MR On-SeT is used in the next section.

\section{$5 \quad$ Results}

The survey findings revealed that from December 2019 to March 2020 the participating OHS managers conducted OHS training sessions where they utilised MR On$\mathrm{SeT}$ with around 540 employees (ranging from 16 years to 68 years, $22 \%$ females and $78 \%$ males) during regular OHS instructions for existing and new employees with different knowledge levels. Also, the application has been presented and tested on management meetings, public events, etc. (Table 1). We received feedback on how the local OHS managers experience the application and the feedback they got from workers of their plant, during and after OHS training sessions. In Table 2, we present the identified and clustered themes and subthemes. 
From the data, two end-user perspectives emerge: 1. the OHS manager as orchestrator, teacher and facilitator of the training, and 2. the trainee as learner in MR. Thus, we report our results based on these perspectives.

\subsection{The occupational safety manager: Teacher, facilitator, and orchestrator}

Because of the multiple roles OHS managers have to perform; several themes directly contribute to the understanding of their needs and experience using $M R O n-S e T$ (see Table 2).

Values: The use of shared learning. Shared Learning for training on occupational safety differs from the current practice, which rather can be described as a formal learning setting. Also, when using MR, a computer mediated communication channel needs to be established to let people share their experiences and experience those of others.

Table 1. Occasions the Occupational Safety Managers used MR On-Set emerging from the question: "At which occasion did you use the safety training already?"

\begin{tabular}{|l|l|l|}
\hline \multicolumn{1}{|c|}{ Theme } & \multicolumn{1}{|c|}{ Participant } & \multicolumn{1}{c|}{ Examples } \\
\hline $\begin{array}{l}\text { Regular Training } \\
\text { Sessions }\end{array}$ & $\mathrm{R} 1, \mathrm{R} 2, \mathrm{R} 4, \mathrm{R} 6$ & $\begin{array}{l}\text { Trainings with safety officers (R4), } \\
\text { Initial HSE training for new employees and during periodical } \\
\text { trainings (R2) }\end{array}$ \\
\hline $\begin{array}{l}\text { Internal Promotions } \\
\text { (Management Level) }\end{array}$ & $\mathrm{R} 3, \mathrm{R} 6$ & $\begin{array}{l}\text { In health and safety committee meeting (R3), } \\
\text { Use after announcement in company newspaper on request of } \\
\text { managers with teams and mixed teams (R3) }\end{array}$ \\
\hline $\begin{array}{l}\text { Promotional and } \\
\text { Marketing Events }\end{array}$ & $\mathrm{R} 1, \mathrm{R} 3, \mathrm{R} 5$ & $\begin{array}{l}\text { Regarding Safety Events (R1), } \\
\text { In meetings of the master craftsmen association of several loca- } \\
\text { tions (R3) }\end{array}$ \\
\hline Not used & $\mathrm{R} 7$ & Not start, yet (R7) \\
\hline
\end{tabular}

Several respondents (R1, R3, R4, R7) described how the use of MR On-SeT in their training sessions is enabling Shared Learning. R4, for example mentions that streaming the image to an additional screen can be employed to allow others to participate in the trainee's experience. Reflection on the shared experience then takes place in the group after the recent session. Using MR On-SeT in this way was confirmed by R3, who stated that the application "provides the basis to start a discussion on the content (safety)". Some respondents (R1, R3) described how they use MR On-Set as a safe space, where trainees can experience hazardous situations without being exposed to a real threat. They use the experiences from MR On-SeT to let the trainees reflect on OHS. This can also affect the way OHS managers will plan training sessions and needs means to carefully mediate the interaction between trainer and the immersed trainee. 
Table 2. Themes and Subthemes based on the Survey Results.

\begin{tabular}{|c|c|c|}
\hline Theme & Subtheme & Examples \\
\hline \multirow{2}{*}{ Content } & Variety & $\begin{array}{l}\text { Respondents noted positively that } M R O n-S e T \text { has a high variety of scenarios } \\
\text { but also suggested further scenarios and other OHS related use cases for } M R \\
O n-S e T \text {. }\end{array}$ \\
\hline & Tailorability & $\begin{array}{l}\text { Respondents requested to be able to adapt the system to local needs but also to } \\
\text { the capabilities of individual trainees and maintain the content based on inci- } \\
\text { dents related to OHS. }\end{array}$ \\
\hline \multirow{2}{*}{$\begin{array}{l}\text { Shared } \\
\text { Learning }\end{array}$} & \begin{tabular}{|l|} 
Technological \\
Enabler
\end{tabular} & $\begin{array}{l}\text { Respondents reported positively on streaming the trainees' field of view to an } \\
\text { additional screen but also mentioned issues while using this option. }\end{array}$ \\
\hline & Reflection & Respondents described how they use $M R O n-S e T$ to start a discussion on OHS. \\
\hline \multirow{2}{*}{$\begin{array}{l}\text { Simulation } \\
\text { Sickness }\end{array}$} & Symptoms & $\begin{array}{l}\text { Symptoms which can be accounted to simulation sickness were reported by the } \\
\text { responding OHS managers. }\end{array}$ \\
\hline & $\begin{array}{l}\text { System } \\
\text { Behaviour }\end{array}$ & $\begin{array}{l}\text { Some OHS managers reported system behaviour of } M R O n-S e T \text { and the Ho- } \\
\text { loLens that can cause symptoms which can be accounted to simulation sick- } \\
\text { ness. }\end{array}$ \\
\hline \multirow{3}{*}{$\begin{array}{l}\text { Learn } \\
\text { through } \\
\text { Play }\end{array}$} & Realism & $\begin{array}{l}\text { We got positive feedback on the visual and auditive realism } M R O n-S e T \text { pro- } \\
\text { vides. }\end{array}$ \\
\hline & Orchestration & $\begin{array}{l}\text { Respondents stated the need to adapt and extend the scenarios to be able to fit } \\
\text { it to their training sessions. }\end{array}$ \\
\hline & Gamification & $\begin{array}{l}\text { Respondents highlighted the playful character, the interactive manner of } M R \\
O n-S e T \text { and its overall attractiveness. }\end{array}$ \\
\hline \multirow{2}{*}{$\begin{array}{l}\text { Enter } \\
\text { Interaction }\end{array}$} & \begin{tabular}{|l} 
Gestural \\
Interaction \\
\end{tabular} & $\begin{array}{l}\text { Some trainees had problems with the gesture-based interaction mode of Mi- } \\
\text { crosoft HoloLens 1. Respondents suggested improving or even replacing it. }\end{array}$ \\
\hline & Scanning & $\begin{array}{l}\text { Respondents reported that aligning the mixed environment with the real world } \\
\text { to be complex and sometimes failed }\end{array}$ \\
\hline
\end{tabular}

There seems to be the desire to actively fill out the role of a mediator of the OHS managers. Therefore, we offer a hybrid computer mediated communication channel, serving as Technological Enabler (streaming option), which needs to be established. R5 reported that the streaming option, i.e., streaming what the immersed trainee is currently experiencing in MR, to an additional screen to allow others (OHS managers and trainees) to follow the immersed trainee, is a positive feature. Three others (R3, R4, R6) felt restrained in terms of actively participating by the issues related to streaming. While R3 reported a too large delay in transmission, which inhibits following the trainees in MR on what they are doing, to directly raise questions (as a Teacher) or guide them through MR On-SeT (being a Facilitator and Motivator), R4 requested a streaming option. The latter might be due to the fact that the respondent did not find the suitable information in the user manual we handed out with the application. Streaming, and therefore Shared Learning, is not just inhibited by the delay of transmission. R6 mentioned that "the operation of AR glasses and [the companies] IT cannot be combined", which relates to the required connection of both devices to a wireless network. Since the local IT-infrastructure does not allow to connect uncertified devices, OHS managers have to administer their own.

The work to make the training work: Enter interaction in MR to set-up the stage: Preparing the training session and interrupting as well as resuming the MR training is a requirement to conduct OHS training with $M R O n-S e T$. As a stand-alone solution (R6), in opposite to former VR and MR solutions which rely on additional hardware to be set up to (e.g., to enable user tracking), MR On-SeT takes MR train- 
ings out of the lab, where technical experts run the hardware and software and transfers this task to non-expert users. These non-expert users then consequently have to Enter Interaction with the system. Since they are new to the use of MR and its prerequisites, the necessary steps needed to run the application and how to trigger them (by interacting with the system) have to be reduced. The Scanning wizard of MR On$\mathrm{SeT}$, a tool to allow the OHS managers to align the virtual content with the real environment, exemplifies this need for simplification, since we attempted not to hide the process' complexity. This unhidden complexity directly affects the OHS managers in the execution of their role as orchestrator: $M R O n-S e T$ requires the OHS managers to prepare the application while pre-orchestrating the training session. In this role, they have to scan the room where the training shall take place. Three Respondents (R1, R2, $\mathrm{R} 4)$ experienced related issues. For example, R1 stated that "Measuring the room is sometimes not easy", while R2 highlighted the insufficient description, "especially for the calibration", in the user manual we handed out.

Some respondents (R1, R3, R4) reported on issues related to resetting the application and its content. Also related to the task of orchestrating a training session, two participating OHS managers ( $\mathrm{R} 1, \mathrm{R} 4)$ recognised, that as soon as the application is sent to the background (described as closed application), the tracking is lost and the application must be reopened. In addition, R3 recognised issues resetting solved hazards.

Orchestration of trainings: Variety in MR scenes: To be able to use MR Content (see Table 2) for different training goals, OHS managers need to have a Variety in different scenarios in which OHS hazards can be experienced by trainees. Variety can be achieved in a fully pre-orchestrated system such as $M R O n-S e T$ to some degree (R4, R5, R7). Still, some participants saw opportunities to increase scene Variety within the application (R2, R3-5). R3 suggested supporting more training scenarios, while R2 and R4 suggested specific scenarios. R4 pointed out the potential "Integration of further scenarios such as a room to hazardous substances...".

The OHS managers' remarks regarding their empowering to orchestrate Content by themselves (e.g., intentionally vary arrangements) are grouped under our Tailorability subtheme. Three respondents (R5, R6, R7) saw the need to tailor MR On-SeT to their own needs by themselves. This was expressed by e.g., requests to "adapt the risks to the plant." (R5). Being able to tailor the Content to specific needs of one training session automatically increases the Variety of experiences trainees can have in MR. Also, the request to customise $M R O n-S e T$ by translating it to the local native language (R2) is related to Tailorability.

Variety at a higher level is represented by the suggestions (R3, R5) of different ways to use MR On-SeT. R3 suggested to offer "More opportunities for interaction (possibly also with the environment)". R5 suggested to allow trainees to take the perspective of someone who is working in an environment whereby they are causing risks to others (e.g., driving a forklift) or are exposed to increased risk (e.g., working in great heights).

The respondents expressed a need for higher flexibility in orchestrating the experience, which highlights that even though, $M R O n-S e T$ is used within one company 
only, there are still local differences, e.g., the need for text-based instruction in the users' language.

\subsection{The trainee: opportunities for learners in MR}

In contrast to the OHS managers, the trainees only have the role of a learner in $M R$ $\mathrm{On}-\mathrm{SeT}$. Since they are the target user group of $M R \mathrm{On}-\mathrm{SeT}$, their role is related to all themes. We condensed the connection to the themes using the statements of the responding OHS managers. We are aware that what OHS managers say about their own experiences has more validity than on what they report on the trainees' experiences. Still, since they supervise trainees in MR, they are capable of sketching the trainees' perspective and needs.

Mediation: Support and experiences in shared learning: Shared Learning is not only important from the OHS managers' perspective, but also for trainees. Being able to see what the immersed trainee is seeing (using the streaming option) allows other trainees to learn in groups. Afterwards they can start a discussion on the topic of occupational safety within the group or with the OHS manager. This Reflection on the recent session contributes to Shared Learning. It therefore might help to foster a Community of Practice, since employees are encouraged to start a dialogue on the topic of OHS and might define a certain safety climate as described by Zohar [31]. Furthermore, trainees in MR might need help when they are lost and indicating the need to rely on a computer mediated communication channel as Technological Enabler. Here the OHS managers can help by following the trainees on the screen. R3 confirmed the need for streaming as a mediated communication channel. However, $\mathrm{s} /$ he stated that the current delay in transmission makes following the trainees in MR time consuming. This delay in the mediated communication forces the trainees and the OHS managers to enter a dialogue on navigation through the MR. This dialogue mainly consists of instructions from the OHS managers (such as simple directional navigation, but also request to show a certain object), who are trying to anticipate the mixed environment, and reactions and call-backs from the trainees trying to align the instructions with the actual mixed environment. Instead of reflecting on the topic of OHS, the trainees hence try to figure out how to interpret the OHS managers' instructions on actions, which the trainees executed around three seconds ago. Experiences users can have in a mediated reality with a delayed transmission were illustrated in a commercial spot by Umeå Energi AB [32].

Mitigating simulation sickness: Simulation Sickness needs to be considered when designing Mixed Learning Environments but also when pre-orchestrating individual training sessions. MR (and VR) experiences are still influenced by this phenomenon. Since trainees are the users of $M R O n-S e T$ who are exposed to the mixed environment, Software Behaviour causing Simulations Sickness and Symptoms of Simulation Sickness form an important, but not exclusive, theme for the trainees. One responding OHS manager (R5) reported that tracking issues during movement can cause Simulation Sickness, while R3 and R4 specify image flickering while moving. Another responding OHS manager (R7) reported how the selected hardware can become a factor in regard to Simulation Sickness. The high weight of the HMD puts too much load on 
the bridge of the nose (via the nose pad of the HMD) resulting in a painful experience. Dizziness or feeling bad after use was reported by R6.

Even though we decided to use MR technology to address the persisting issue of Simulation Sickness, respondents report on this phenomenon. We selected a less immersive display technology than VR, expecting that constantly perceiving the real environment might reduce the effects of visual causes. Still, the problem of Simulation Sickness is highly complex and relates to more than one cause. With current hardware, we recommend that users are exposed to MR less than 30 minutes (according to own experience, but also reported by R3).

To improve tracking, which is not just relying on the registered room dimensions but also on colour information, unicoloured walls and floors should be avoided. Offering pre-designed posters, which can be printed right before the use, can help to address this issue. Furthermore, OHS managers can reduce the time each trainee is exposed to MR (and consequently to potential system behaviour which causes simulation sickness) when training groups by letting each of them only solve a certain number of hazards and then hand-over the device to the next trainee. This might support Shared Learning. While Simulation Sickness mostly is an unwanted phenomenon, it could be also employed to intensify experiences such as operating heavy machinery [33]. Issues with streaming the trainees (in MR) field of view to an additional screen need to be addressed properly.

First steps in MR: Enter interaction: Two OHS managers (R1, R2) stated that MR On-SeT is "very interactive". However, many trainees are novice users of MR and Gestural Interaction, who need a low threshold to Enter Interaction. Based on the feedback of respondents, there is need of better designing the Gestural Interaction of $M R \mathrm{On}-\mathrm{SeT}$, since interacting with the device caused problems, four responding OHS managers (R3, R5, R6, R7) reported. All of them noted that the gesture-based interaction poses higher level of complexity for some trainees, mainly specified as being difficult to execute the drag and drop gesture. R6 for example, specified that drag and drop "is extremely difficult for some [trainees]". Two OHS managers (R1, R5) proposed to improve on the Gestural Interaction by simplifying it ("Making it easier to click on the danger points", R1) or by offering an alternative interaction mode ("For beginners use controllers", R5).

Motivation in formal learning settings: Learn through play in MR: Learn through Play contributes to occluding the formal learning character of $M R O n-S e T$ and increases the trainees' motivation to engage in the training. MR On-SeT can be placed in the Most Engaging OHS training category defined by Burke et al. [27].

Three responding OHS managers (R1, R2, R3) reported directly on its attractiveness while R4, in addition, mentioned that "Working with the app was more interesting for everyone than other types of instruction". Furthermore, R1, R4 and R7 mentioned the playful character of MR On-SeT. Playfulness in combination with attractiveness underlines the potential of Gamification for MR On-SeT. Gamifying formal learning approaches might lead to a more informal way of learning, which than reflects the most common way of acquiring knowledge in the work environment.

Responding OHS managers requested to Orchestrate the scenarios. Being able to adapt and create scenarios based on local requirements or a special focus of a training 
session can be used to keep up the element of surprise for the trainees, since the application will never be exactly the same. Besides increasing the Variety, it also can be used to increase the degree of Realism, since virtual copies of real workplaces, the trainees are actually working in, can be created. Two responding OHS managers (R3, R4) reported that MR On-SeT has a high degree of Realism, while one (R3) positively mentioned the use of scenario-typical sounds, such as by-passing floor-borne vehicles in the manufacturing scenario, we recorded on-site. A high degree of Realism may enable trainees to easier transfer what they just experienced to real work life problems.

Discover new perspectives on safety: Content shapes experiences: The Content is important to provoke trainees to actively participate in training session. The reported Variety of MR On-SeT (by R4, R5, R7) in the scenarios and the scenarios suggested by responding OHS managers (R2, R3, R4, R5) allow trainees to explore different areas representing typical work environments. Hence, $M R O n-S e T$ can be used to awake the human urge to discover unknown things. Besides increasing motivation and arousing the trainees' curiosity, there also is a serious element: Many workers spend little time in other departments, but often use the walkways through it (e.g., to prevent getting out in the winter). In these cases, they are exposed to specific hazards of this area. If trained properly, they also can contribute to the safety of others by identifying and reporting OHS hazards.

\section{Discussion}

Addressing hardware limitations to increase the user experience. R5 requested to be able to use the application in "any environment (not specially in a gloomy room)". This is a problem related to see-through displays used in HoloLens. In a too brightly luminated environment, the maximum display brightness might not be sufficient to enable users in MR to fully see the virtual objects. Therefore, we suggested to slightly dim the lights in the room the training should take place. Another approach to address this problem could be using brighter displays, which than might cause eyestrain (potentially causing Simulation Sickness) or even damage the eyes. Vasilevska et al. [34] discuss how to reduce that eyestrain by offering design suggestions to reduce screen brightness for VR-HMDs. They found that reducing display brightness and using so-called night-modes (where white interface-backgrounds are inverted) is excepted by the user and can help reducing eyestrain. Transferring the concept of night-mode to mixed environments, darker (passive) background objects might already reduce the overall brightness. Also, to further increase visibility additional shades (e.g., similar to the shades of the Epson Moverio BT-350 HMD) could be designed which can be put on top of the see-through display in bright environments, to be able to reduce display brightness.

While designing the application we were aware of the small field of view and tried to address this in our design. We reduced the space, where interactable objects (hazards) are placed, to two of the four walls of the room. Still, respondents reported the small field of view to be problematic. One approach to reduce this might be distorting 
the image close to the outer boarders of the field of view, similar to side mirrors of some heavy goods vehicles or urban buses. Designers need to trade-off the area of distortion and whether the degree of distortion follows the path of any polynomial, which then smoothly fades from no distortion to increased distortion, or there is one pre-defined degree of distortion in this area of distortion. As this small field of view especially limits peripheral visual perception, simple hardware solutions, such as suggested by Xiao and Benko [32] where a low-resolution LED display is placed around the lenses of VR glasses, could be applied as well.

Trainer empowerment: According to Bowers [35] management overheads, such as the OHS mangers experience when preparing $M R O n-S e T$, which result in extra work might "be reason enough for abandoning technologies" [35]. While Bowers in 1994, argues to increase available support, for MR technologies, which depicts a completely different technology compared to the network of stationary computers Bowers investigated on, today, it rather should be: Decrease of necessity of support whenever it is possible. Computers, laptops, and even smartphones and tablet-PCs still not require to actively interact with the physical environment and, therefore, run within relatively stable frame conditions, where support can be supplied, even over long-distance. MR in contrast can be understood as the opposite: The unconditional turn to employing the environment for the purpose of providing a (semi-) virtual environment. The mobile character of the Microsoft HoloLens 1 and MR On-SeT therefore represent a new way technology is used at the workplace. Since every physical room MR On-SeT can be used in differs, the amount of not controllable frame conditions multiplies.

Bowers demand that those who are involved in the maintenance of a technical system (here the OHS managers) should become "heterogenous engineers" also could help to overcome the reported problems, which are related to scanning, resetting, and streaming. This seems to be problematic for a mobile solution, which adds to a set of training tools, such as MR On-SeT. First, the ubiquity of computing with today's mobile technology moves complex systems from professional spaces, where experts operate them, to the less experienced end-users (who want to use the technology as a tool, rather than experimenting with it). Second. the responding OHS managers seem to expect $M R \mathrm{On}-\mathrm{SeT}$ to be working out-of-the-box, rather than need to be educated to make it work. There might be a large discrepancy in the skillset different OHS managers bring along, making it impossible to assume a common level of technological skills.

The need for a Technological Enabler is exemplified in the issue of connecting $M R$ $\mathrm{On}-\mathrm{SeT}$ to a wireless network to use the streaming option and offer Shared Learning. There is the need to empower the OHS managers to establish this streaming option without engaging with complex network administration tasks. The streaming option set-up should reflect the technical skills of the OHS managers. It might be fruitful to allow MR On-SeT to open a wireless network, to which other devices can be connected. This reflects the usual way of connecting a laptop, tablet-PC or smartphone to a network, which might reduce complexity. To allow controlling the live-streaming option and to empower the OHS managers, an interface should be added to MR On- 
$\mathrm{SeT}$, in which the users can activate the wireless network and are guided through the process of connecting another device they want to stream to.

Offering variety by live-orchestration and new learning experiences: The provided Variety of scenes enabled OHS managers to understand the potential of MR On$\mathrm{SeT}$ and inspired them to request more scenes (see theme Content). Still, preorchestrated systems might quickly reach boundaries, which then might affect the perceived benefit, since the Mixed Learning Environment rapidly becomes boring for the trainees. In contrast, pre-orchestrated systems might better make sure that company guidelines are respected. One promising approach to address the effect of wearingoff is offering live-orchestrating selected virtual objects (or avatars), such as Koller et al. [16] propose.

Addressing the potentials and risks of Mixed Learning Environments might already affect how systems are orchestrated, since the pre-orchestrated elements of a mixed environment can drastically influence how people perform tasks in MR. The combination of real and virtual environment can lead to a diminished distinction of when the MR and when reality is experienced [36]. According to Brey [37], there is the responsibility for designers to consider and prevent ethical questionable actions (e.g., active violence against a non-playable character) and the general depiction of ethical questionable content in MR (e.g., as proposed by Van Wyk and De Villiers [21] or by letting trainees experience accidents by themselves). Live-orchestrating can amplify this responsibility since live-orchestrators can alter the perceived environment of others. They might be able to annoy users in MR, e.g., by sudden changes in the setup, but also can have an extensive influence on what the users in MR can and cannot see. Live-orchestrators might even perform violent actions against users in MR, while perceiving them as virtual characters. While live-orchestration, therefore, can be a powerful tool to influence MR users' acting and thinking it is one potential solution to keep up the element of novelty. Currently, $M R O n-S e T$ is perceived as novel, since both, HMD and MR are not widely spread, neither at work nor in private life. Constantly introducing new hardware or hardware add-ons in the field of serious applications will probably be too ambitious. Still, to reach high levels of motivation and even pleasant anticipation, keeping novelty in $M R O n-S e T$ on the long run appears to be important. Besides live-orchestration, designers might want to include Easter Eggs (i.e., hidden features, mainly entertaining), or dynamically change arrangements in the mixed environment.

Besides requesting live-orchestration-like features, respondents recognised new (related) possibilities to make use out of the potential of $\mathrm{MR} \mathrm{On}$-SeT by adding Variety at a higher level. The proposed increasing of (also non-hazardous) interactable virtual objects can be interpreted in two ways: First, currently only the virtual objects that are part of a hazardous situation can be manipulated. It might add to the perceived Realism if trainees also can, for example, grab a pencil, which is lying on a desk, as they could in the real world. Their behaviour might even create new hazardous situations (e.g., when dropping a pencil). Second, allowing trainees to manipulate more objects in their environment might occlude the hazards and therefore increase overall complexity of the training, which can be a mean to create new experiences without live-orchestration. This might introduce a design tension defined by the flow theory of 
Csikszentmihalyi [38]: more complex systems might further increase the threshold to initially engage, since unexperienced and cautious trainees might feel overwhelmed by the complexity of the system, while too simple systems might bore trainees.

Experiencing working in highly risky environments has been suggested. Bosche et al. [17] and Yabuki et al. [20] for example already describe how working in great heights can be simulated. Another example of experiencing risky work environments has been demonstrated by Van Wyk and De Villiers [21] where trainees can observe virtual colleagues (i.e., a non-playable characters) who ignore safety rules and experiencing very severe consequences (e.g., being pulled into a running machine after trying to remove an object from it). Some consequences of accidents might be serious but even be experienced by the trainees themselves. Since these experiences then are intentionally caused, users might perceive this as interference with their physical integrity (as described by Brey [37]). The same accounts for intensifying experiences by employing Simulation Sickness [33]. It has to be kept in mind that some users respond very heavy on System Behaviour which cause Simulation Sickness. To address this ethical dilemma, designers could offer an option, where users can decide on their own how much intensifying Simulation Sickness they want to experience. Another solution, proposed by Freiwald et al. [39], would be assessing each users susceptibility to Simulation Sickness by using the Cybersickness Susceptibility Questionnaire (CSSQ), which aims to predict the likeliness a user is affected by Simulation Sickness. The CSSQ could be included into VR or MR applications intentionally causing Simulation Sickness.

Furthermore, mobile systems mainly are administrated by users on site, this directly influences how the orchestration of the mobile applications can look like. Any orchestration of MR systems needs to take the fact into account, that non-expert users (in terms of MR) need to set up and control the behaviour of the mixed environment by themselves. This poses the challenge of simplifying setting up and maintaining a system.

Introducing altered environments for learning at work heavily can affect the way of working but also the general work-life. Some replies of the respondents indicate that MR On-SeT has the potential to pose a disruption, since it can change how OHS training takes place in future. While disruptions can mean an interesting new way of working or learning (as it was reported for MR On-SeT), they always include the risk of causing affected workers to reject changes [35], since it might expose their way of doing things to their supervisor which could make them feel observed all the time.

Translucency of new interaction modes and fallback solutions: The theme Enter Interaction reveals that there are difficulties interacting with the system for individual users. This could be due to missing translucency of the gestural input, which possibly lead to request a change in the interaction paradigm. As described by Ebling et al. [40], translucent (socio-technical) systems need to "expose critical aspects [...] while hiding noncritical details to preserve usability".

Problems with the input modes and the suggestions to improve interaction indicate the gap between the potential of MR and its understanding by users and therefore missing translucency. In the new version of Microsoft HoloLens, HoloLens 2, a concept to increase translucency towards the input-gestures has been established. As soon 
as the HoloLens 2 detects a gesture, it shows a 3D model of a hand, which demonstrates how to execute the gesture properly.

It is an interesting fact that companies and researchers, working with HMDs, include gesture-based interaction and naming this mode of interaction "natural interaction". In serious applications, established (hence, known) interaction modes are understood to simplify the interaction (such as controllers of video game consoles). They might therefore be perceived as mean to address the need for a translucent system. Even gestures, which are understood as known by a large part of the population, such as dragging objects on a touchscreen, are not guaranteed to be understood by all users, as shown for elderly users by Mihajlov et al. [41]. The particular request of controllers might not just be related to problems with executing gestures correctly but also with effects like haptic uncanny valley [42], i.e., that the haptic sense often still is excluded from the experience, while being important for human perception.

Translucency to some extend overlaps with the change of user tasks due to the lack of direct expert support: Respondents mentioned they sometimes had problems to run the scanning wizard successfully, which is a basic requirement to execute MR OnSeT. A more translucent set-up-wizard, which for example enables the user to better understand why the application needs to scan the environment and which measures can be undertaken to increase the probability of success, can reduce the complexity of setting up the mixed environment.

\section{Design Implications}

In this section implications for designing MR training systems are presented. The implications are based on the themes.

Awakening the discoverer: Designers should reflect on how to keep up the element of novelty in their mixed learning environment (Theme Learn through Play, subtheme Gamification). If trainees have the feeling, there still is something left to discover, even though they explore the mixed environment yet again, they might be more motivated to actively participate in the training due to their curiosity. Besides serious content, which is hard to find, Easter Eggs or other entertaining features could be included. Furthermore, if possible, it is advisable to work with the element of novelty of a technology, to promote the Mixed Learning Environment.

Support playfulness in learning - 'Informalise' formal learning settings: We suggest designing an application like $M R O n-S e T$ towards user engagement but in a realistic way. Based on our experience and as reflected in Theme Learn through Play (subthemes Realism and Gamification), enabling users to explore and try things out in a playful way, already adds to the entertaining character. This might change how trainees perceive the character of OHS training from formal towards informal learning (which reflects how learning often takes place at work). Furthermore, designers should try to create a training climate in which playfulness is not inhibited by observing trainees or the trainer (as described by Shared Learning), to keep the immersed trainee experimenting and reflecting. 
Support non-it users to orchestrate and create variety: The design for a training application, which is used on a world-wide scale in a formal setting, such as MR On$\mathrm{SeT}$, requires adaptions to local specialities. Therefore, we argue for designing a (semi-) flexible orchestration concept (Theme Content, subtheme Tailorability and Theme Learn through Play, subtheme Orchestration) that would allow trainers to address these local needs and adapt the application to their particular workflow. Carefully offering flexibility in the design of the mixed environment should include preorchestrated content while providing the possibility to trainers to orchestrate for local specialities. A special focus when analysing the context of use should be on the need for flexible orchestration. Furthermore, the analysis should ensure that the context of use is fully understood, and relevant stakeholders (enabler) could verbalise their requirements adequately. Finally, designers need to be aware of the potential ethical issues related to live-orchestration and prevent misuse by design.

Translucency lowers the threshold to engage: To allow users of MR training systems to understand complex system behaviour (Theme Enter Interaction, subtheme Gestural Interaction), the system needs to (e.g., visually) reflect the users' activity to a certain extend. While trainees are in the Mixed Learning Environment, they should not be forced to understand how to interact with the system and why the system reacts in a certain way. They rather should focus on understanding the topic, in our case OHS safety, by engaging with the mixed environment. It is advisable to carefully include feedback mechanisms, which inform the user how to correctly interact with the system. Designers should keep in mind that translucency is not just informing the users on wrong or unclear input but is a powerful tool to introduce users to an interaction concept in an informal 'learning by doing' way.

Design for the itinerant trainer: Technological progress allows to design fully mobile HMDs such as Microsoft HoloLens, which therefore also can operate without additional infrastructure (e.g., for rendering). While this has many advantages (e.g., no cables) it also will transfer responsibility to the user (e.g., to prepare the physical environment without assistance by an expert), which increases the threshold to Enter Interaction to pre-orchestrate the MR learning environment. Designers must account for that in their design solutions, for example, by including wizards or similar concepts to guide the user through setting up the system.

Disrupt with mixed learning environments: Introducing MR into learning practice can be disruptive, as it not just provides a new tool to use but changes the way employees perceive the work environment and also how they work. Designers should reflect this in their concepts, for example, by mainly working with realistic 3D models (in contrast to Shamsudin et al. [7]) and designing for self-descriptiveness and learnability. They need to place their design into the current understanding of digital environments and be aware that they might shape this understanding with their design.

\section{Conclusion}

In this paper, we present MR On-SeT, a MR technology-based OHS training enabling trainees to explore hazardous situations, without being exposed to real-life 
threats. MR On-SeT offers a space in which playful learning takes place but also allows to interact with the immersed trainee.

We conducted an expert survey to collect early experiences directly after the rollout of the system. Respondents of the presented survey identified the high potential of the solution by requesting the possibility to orchestrate the scenes by themselves to integrate current topics, which would allow a training tailored to the local needs. The respondents reported high levels of engagement and perceived MR On-SeT as an extension to the tools they can use for OHS training. Also, they highlighted the need for adaptable scenarios, such as tailoring hazardous situations to their specific (local) training program. Some users (trainers as well as trainees) experienced technical issues. Furthermore, some OHS managers reported that especially unexperienced trainees have problems with the interaction concept. Additionally, the OHS managers already include the solution in the regular OHS training sessions.

Based on the study results, we propose design implications crucial to the design of a MR learning application.

In future work, the themes identified with the inductive approach of our thematic analysis can be used for applying a deductive approach in a follow-up study. We want to further investigate on the potential of MR On-SeT to enable occupational managers to understand the trainees' experiences while they are exposed to MR. This includes the problems trainees have while working in MR, but also the challenge to assess the trainees' level of knowledge in the field of OHS. To better support the communication between the trainer and the trainee, we want to investigate how to offer out-of-the-box solutions, which would allow the trainer to understand the trainee's current situation and offer software tools to actively support the immersed trainee.

\section{Acknowledgements}

We thank Robert Bosch GmbH, Abstatt, Germany for the close cooperation and opportunity to conduct this project.

\section{References}

[1] D. Liu, K. K. Bhagat, Y. Gao, T.-W. Chang, and R. Huang, "The Potentials and Trends of Virtual Reality in Education," in Virtual, Augmented, and Mixed Realities in Education, J. Liu, Dejian and Dede, Chris and Huang, Ronghuai and Richards, Ed. Springer, Singapore, 2017, pp. 105-130. https://doi.org/10.1007/978-981-10-5490-7 7

[2] M. A. Gigante, "Virtual Reality: Definitions, History and Applications," in Virtual Reality Systems, Elsevier, 1993, pp. 3-14. https://doi.org/10.1016/b978-0-12-227748-1.50009-3

[3] World Economic Forum, "The Future of Jobs Report 2018 | World Economic Forum," 2018. Accessed: Apr. 14, 2020. [Online]. Available: https://www.weforum.org/reports/thefuture-of-jobs-report-2018.

[4] P. Diegmann, M. Schmidt-Kraepelin, S. Eynden, and D. Basten, "Benefits of augmented reality in educational environments-A systematic literature review," in Wirtschaftsinformatik Proceedings 2015, 2015, vol. 3, no. 6, pp. 1542-1556. 
[5] A. Setiawan, F. Agiwahyuanto, and P. Arsiwi, "Paper-A Virtual Reality Teaching Simulation for Exercise During Pregnancy A Virtual Reality Teaching Simulation for Exercise During Pregnancy," Int. J. Emerg. Technol. Learn., vol. 14, no. 01, pp. 34-48, Jan. 2019, https://doi.org/10.3991/ijet.v14i01.8944.

[6] J. E. Márquez Díaz, C. Andrés, D. Saldaña, C. Alberto, and R. Avila, "Virtual World as a Resource for Hybrid Education,” Int. J. Emerg. Technol. Learn., vol. 15, no. 15, pp. 94 109, Aug. 2020, doi: 10.3991/ijet.v15i15.13025.

[7] N. M. Shamsudin, N. H. N. Mahmood, A. R. A. Rahim, S. F. Mohamad, and M. Masrom, "Virtual Reality Training Approach for Occupational Safety and Health: A Pilot Study," Adv. Sci. Lett., vol. 24, no. 4, pp. 2447-2450, Sep. 2018, https://doi.org/10.1166/asl.2018. 10977.

[8] F. Ke, S. Lee, and X. Xu, "Teaching training in a mixed-reality integrated learning environment," Comput. Human Behav., vol. 62, pp. 212-220, Sep. 2016, https://doi.org/10. 1016/i.chb.2016.03.094.

[9] A. Neal, M. A. Griffin, and P. M. Hart, "The impact of organizational climate on safety climate and individual behavior," Saf. Sci., vol. 34, no. 1, pp. 99-109, 2000, https://doi. org/10.1016/s0925-7535(00)00008-4.

[10] World Health Organization, "Summary report of the twelfth session of the joint ILO/WHO Committee on Occupational Health, Geneva 5-7 April 1995. Geneva," 1995.

[11] J. Mullen, E. K. Kelloway, and M. Teed, "Employer safety obligations, transformational leadership and their interactive effects on employee safety performance," Saf. Sci., vol. 91, pp. 405-412, 2017, https://doi.org/10.1016/j.ssci.2016.09.007.

[12] G. Lawson, E. Shaw, T. Roper, T. Nilsson, L. Bajorunaite, and A. Batool, "Immersive virtual worlds:Multi-sensory virtual environments for health and safety training," Nottingham, 2019.

[13] P. Milgram, H. Takemura, A. Utsumi, and F. Kishino, "Augmented reality: a class of displays on the reality-virtuality continuum," in Telemanipulator and Telepresence Technologies, Dec. 1994, vol. 2351, pp. 282-292, https://doi.org/10.1117/12.197321.

[14] B. Koleva et al., "Orchestrating a mixed reality performance," in Conference on Human Factors in Computing Systems - Proceedings, 2001, pp. 38-45, doi: 10.1145/365024.36 5033.

[15] M. Koller, S. F. Rauh, G. Meixner, A. Lundström, and C. Bogdan, "Designing for Orchestration in Mixed and Virtual Reality: Challenges and Best Practices," in Proceedings of the 11th Nordic Conference on Human-Computer Interaction: Shaping Experiences, Shaping Society, Oct. 2020, pp. 1-3, https://doi.org/10.1145/3419249.342008 $\underline{0}$.

[16] M. Koller, S. F. Rauh, A. Lundström, C. Bogdan, and G. Meixner, "Continuous Interaction for a Virtual Reality Exposure Therapy System.," in 2020 IEEE International Conference on Healthcare Informatics, Dec 2020.

[17] F. Bosché, M. Abdel-Wahab, and L. Carozza, "Towards a Mixed Reality System for Construction Trade Training," J. Comput. Civ. Eng., vol. 30, no. 2, Mar. 2016, https://doi. org/10.1061/(asce)cp.1943-5487.0000479.

[18] R. Eiris Pereira, M. Gheisari, and B. Esmaeili, "Using panoramic augmented reality to develop a virtual safety training environment," in Construction Research Congress 2018: Safety and Disaster Management - Selected Papers from the Construction Research Congress 2018, 2018, vol. 2018-April, pp. 29-39, https://doi.org/10.1061/9780784481288. 004.

[19] R. Eiris, M. Gheisari, and B. Esmaeili, "PARS: Using Augmented 360-Degree Panoramas of Reality for Construction Safety Training," Int. J. Environ. Res. Public Health, vol. 15, no. 11, p. 2452, Nov. 2018, https://doi.org/10.3390/ijerph15112452. 
[20] N. Yabuki, P. Limsupreeyarat, and T. Tongthong, "Collaborative and Visualized Safety Planning for Construction Performed at High Elevation," in Cooperative Design, Visualization, and Engineering, 2010, pp. 282-285, https://doi.org/10.1007/978-3-642$16066-0 \quad 42$.

[21] E. Van Wyk and R. De Villiers, "Virtual reality training applications for the mining industry," in Proceedings of AFRIGRAPH 2009: 6th International Conference on Computer Graphics, Virtual Reality, Visualisation and Interaction in Africa, 2009, pp. 5364, https://doi.org/10.1145/1503454.1503465.

[22] B. G. Witmer and M. J. Singer, "Measuring presence in virtual environments: A presence questionnaire," Presence Teleoperators Virtual Environ., vol. 7, no. 3, pp. 225-240, 1998, https://doi.org/10.1162/105474698565686.

[23] J. J. LaViola, "A discussion of cybersickness in virtual environments," ACM SIGCHI Bull., vol. 32, no. 1, pp. 47-56, Jan. 2000, https://doi.org/10.1145/333329.333344.

[24] E. A. Boyle et al., "An update to the systematic literature review of empirical evidence of the impacts and outcomes of computer games and serious games," Comput. Educ., vol. 94, pp. 178-192, Mar. 2016, https://doi.org/10.1016/j.compedu.2015.11.003.

[25] R. Nørgård, N. Whitton, and C. Toft-Nielsen, "Playful teaching between freedom and control: exploring the magic circle in higher education," in SRHE International Annual Research Conference, Dec. 2016.

[26] N. Whitton, "Playful learning: Tools, techniques, and tactics," Res. Learn. Technol., vol. 26, May 2018, doi: 10.25304/rlt.v26.2035.

[27] M. J. Burke, S. A. Sarpy, K. Smith-Crowe, S. Chan-Serafin, R. O. Salvador, and G. Islam, "Relative effectiveness of worker safety and health training methods," Am. J. Public Health, vol. 96, no. 2, pp. 315-324, Feb. 2006, https://doi.org/10.2105/ajph.2004.059840.

[28] Microsoft Corporation, "Gaze and commit - Mixed Reality | Microsoft Docs." Accessed: Apr. 17, 2020. [Online]. Available: https://docs.microsoft.com/en-us/windows/mixedreality/gaze-and-commit.

[29] C. Maestas, "Expert surveys as a measurement tool: Challenges and new frontiers," in The Oxford Handbook of Polling and Polling Methods, Oxford University Press, 2015, pp. 583-608.

[30] V. Braun and V. Clarke, "Using thematic analysis in psychology," Qual. Res. Psychol., vol. 3, no. 2, pp. 77-101, Dec. 2006, doi: 10.1191/1478088706qp063oa.

[31] D. Zohar, "Safety Climate: Conceptualization, Measurement, and Improvement," in The Oxford Handbook of Organizational Climate and Culture, B. Schneider and K. M. Barbera, Eds. Oxford University Press, 2014, pp. 317-334. https://doi.org/10.1093/ oxfordhb/9780199860715.013.0017

[32] umeaenergi, "Living with lag - An oculus rift experiment," youtube, 2014. https://www.youtube.com/watch?v= fNp37zFn9Q (accessed Sep. 22, 2020).

[33] S. Von Mammen, A. Knote, and S. Edenhofer, "Cyber sick but still having fun," in Proceedings of the ACM Symposium on Virtual Reality Software and Technology, VRST, Nov. 2016, pp. 325-326, https://doi.org/10.1145/2993369.2996349.

[34] K. Vasylevska, H. Yoo, T. Akhavan, and H. Kaufmann, "Towards eye-friendly VR: How bright should it be?," in 26th IEEE Conference on Virtual Reality and 3D User Interfaces, VR 2019 - Proceedings, Mar. 2019, pp. 566-574, https://doi.org/10.1109/vr.2019.8797752.

[35] J. Bowers, "The work to make a network work: Studying CSCW in action," in Proceedings of the 1994 ACM Conference on Computer Supported Cooperative Work, CSCW 1994, Oct. 1994, pp. 287-298,. https://doi.org/10.1145/192844.193030

[36] M. Slater et al., "The Ethics of Realism in Virtual and Augmented Reality," Front. Virtual Real., vol. 1, p. 1, Mar. 2020, doi: 10.3389/frvir.2020.00001.

[37] P. Brey, "The ethics of representation and action in virtual reality," Ethics Inf. Technol., vol. 1, no. 1, pp. 5-14, 1999, doi: 10.1023/A:1010069907461. 
[38] M. Csikszentmihalyi, Flow and the Foundations of Positive Psychology: The Collected Works of Mihaly Csikszentmihalyi, 1st ed. Springer Netherlands, 2006.

[39] J. P. Freiwald, Y. Göbel, F. Mostajeran, and F. Steinicke, "The Cybersickness Susceptibility Questionnaire: Predicting Virtual Reality Tolerance," in Proceedings of the Conference on Mensch Und Computer, 2020, pp. 115-118, doi: 10.1145/3404983.3410022.

[40] M. R. Ebling, B. E. John, and M. Satyanarayanan, "The Importance of Translucence in Mobile Computing Systems," ACM Trans. Comput. Interact., vol. 9, no. 1, pp. 42-67, Mar. 2002, https://doi.org/10.1145/505151.505153.

[41] M. Mihajlov, M. Springett, and E. Lai-Chong Law, "Learning to Drag: The Effects of Social Interactions in Touch Gestures Learnability for Older Adults," in Proceedings of the 2019 ACM International Conference on Interactive Surfaces and Spaces, Nov. 2019, pp. 205-215, https://doi.org/10.1145/3343055.3359716.

[42] C. C. Berger, M. Gonzalez-Franco, E. Ofek, and K. Hinckley, "The uncanny valley of haptics," Sci. Robot., vol. 3, no. 17, Apr. 2018, doi: 10.1126/scirobotics.aar7010. https:// doi.org/10.1126/scirobotics.aar7010

\section{Authors}

Sebastian Felix Rauh is a research associate at Heilbronn University in Heilbronn, Germany. His current research focuses on supporting and training workers using Mixed Reality technologies.

Marius Koller is a research associate at Heilbronn University in Heilbronn, Germany. His current research focuses on the orchestration of virtual environments.

Cristian Bogdan is Associate Professor at KTH Royal Institute of Technology in Stockholm Sweden. His interests include designing technologies for learning in communities of practice.

Olga Viberg is Associate Professor in Media Technology at KTH Royal Institute of Technology in Stockholm, Sweden. Her research focuses on the design and evaluation of technology-enhanced learning solutions in formal and informal learning settings.

Philip Schäfer is a research associate at Heilbronn University in Heilbronn, Germany. His research interests include virtual reality and how human behaviour can be influenced with subliminal stimuli in this environment.

Gerrit Meixner is Research Professor for Human-Computer Interaction at Heilbronn University in Heilbronn, Germany and Affiliated Professor at KTH Royal Institute of Technology in Stockholm, Sweden. His research focuses on usability engineering and innovative interaction technologies.

Article submitted 2020-11-05. Resubmitted 2021-01-05. Final acceptance 2021-01-07. Final version published as submitted by the authors. 